\title{
The management of injuries to young teeth ${ }^{*}$
}

\author{
William E. Brown, D.D.S., M.S.†
}

Injuries to primary and young permanent teeth from simple falls around the home and school, automobile accidents, and various contact sports may result in disfigurement and disruptive changes in occlusion and alignment of the teeth. Whereas many injuries can be prevented with simple protective devices, others cannot be avoided. It is the purpose of this presentation to discuss the available preventive measures and the diagnosis and treatment of a variety of injuries.

\section{Prevention of injuries}

For years seat belts have been standard equipment in airplanes. Much more recently seat belts have become optional equipment in automobiles, and now some thirty States have adopted statutes making seat belts mandatory for the cars of the future. It has been demonstrated conclusively that these seat belts will reduce both the number and the severity of injuries, particularly those which occur around the face and the mouth.

In the United States a large percentage of injuries from football and hockey occur to the mouth and teeth. Studies have indicated that these injuries can be reduced markedly by the wearing of suitable mouth guards. In 1962, the largest high school athletic association

* Presented at the 18th Australian Dental Congress, Melbourne, February-March, 1967.

† Professor of Dentistry and Associate Director, W. K. Kellogg Foundation Institute: Graduate and Postgraduate Dentistry, The University of required all high school football players under its jurisdiction to wear mouth guards both during practice and in regular games. Such protectors have been worn by boxers and some football players for many years. Although these appliances afforded reasonable protection, they were bulky and speech with them was difficult. Hence, they were rejected by many athletes. The recent use of latex rubber and thermoplastic vinyl resin has reduced some of the earlier problems. According to Godwin, (1) "the final criteria for protection of the teeth depend on a minimum thickness material for comfort and a maximum degree of fit of the appliance, which will distribute a pointed blow over the entire dental arch." He states further, "that in contact sports, athletes should not wear any removable appliances. Impressions for mouth gaurds should be taken with such appliances removed. If an athlete is em. barrassed about the appearance of missing anterior teeth (thermoplastic vinyl resin is transparent), the impression can be made with the removable appliance in the mouth, and the removable teeth involved can be painted into the mouth guard with fast-cure acrylic. Orthodontic appliances which are fixed to the teeth need not be removed. An impression can be made with these in place, and then the appliance can be blocked out on the model so that a mouth guard will fit over them."

(1) Godwin, W. C.-A simplifled mouth protector technic. II. J. Michigan D. A., 44: 7, 227-234 (July-Aug.) 1962. 
The vinyl mouth protector is constructed in the following fashion:

(1) Secure an impression of the maxillary arch and make a model,

(2) Centre the model on a hollow wooden box, with small holes in the surface, which is attached to a source of vacuum,

(3) Soften a sheet of thermoplastic vinyl resin in boiling water until its surface is tacky and position over the model,

(4) Turn on the source of vacuum and allow it to run for two minutes; the vacuum will pull the plastic sheet tightly and uniformly over the model,

(5) Allow the material to cool on the model for one minute,

(6) Remove the model and material from the vacuum box and chill in cold water, and

(7) Remove the mouth guard from the model and trim the palate and flanges with curved scissors.

The complete technique has been described by Godwin. ${ }^{(1)}$

\section{Injuries to primary incisors}

A blow to the primary incisors of a preschool child may result in discolouration of the offended tooth, coronal fracture, pulpal degeneration, displacement, or avulsion. Colour change by itself means very little other than that the tooth has received a blow and that red blood cells have migrated from their normal channels into the dentinal tubules. Not infrequently the pulp will degenerate, either from the severity of the blow itself or from extensive internal or external resorption of the root. In this event, the tooth usually is extracted.

A tooth which has been driven apically usually will re-erupt and should not be repositioned mechanically. A tooth which has been displaced into a crossed-bite relationship should be repositioned mechanically and splinted in place. An avulsed incisor, or an incisor which has been extracted, usually is not replaced. Spaces created in the anterior segments of the primary dentition rarely close. When closure does take place, it is usually because of a space discrepancy problem, and closure results because of an unwinding of the crowded incisors. Even in this situation, the cuspid to cuspid distance rarely changes. Some clinicians feel that primary incisors should be replaced in order to restore asthetics and prevent psychological disturbances and in order to prevent deleterious speech habits. Studies are not available to support these contentions.

Coronal fractures of primary incisors usually require no treatment other than stoning and polishing to avoid irritation of the tongue and lips. If the pulp has been exposed by the fracture, and this occurs rarely, it may be treated by pulpal capping or pulpotomy. Following this therapy, the tooth may be restored with an acrylic jacket or a preformed stainless steel crown.

Periodic X-ray examinations should be secured of any tooth which has received a blow in order to detect promptly evidence of pulpal death. Pulpal testing with electrical instruments, ice, or heat, is unreliable for primary teeth. Parents should be cautioned that pulpal death is a possibility at any time following a blow to a primary incisor.

\section{Injuries to young permanent incisors}

An injury to a young permanent incisor is generally much more significant than a similar injury to a primary incisor. A coronal fracture or discolouration are highly disturbing to a child and his parents-to say nothing of the severe psychological trauma evoked by the avulsion of a permanent tooth. Any blow to a young permanent incisor should be regarded as serious unless proven otherwise, and thorough diagnosis and treatment should be employed promptly. The ancient custom of deferring any treatment until the child reaches 17 or 18 years of age should be discarded.

\section{Emergency treatment}

Whenever a child has received a severe enough blow to an incisor to produce a coronal fracture, mobllity, or displacement, he should be seen by his dentist within the day. The area of injury should be examined clinically to determine the extent of the injury to the hard and soft tissues; $X$-ray examinations should be secured of the involved teeth as well as of the adjacent and opposing teeth in order to detect such things as root fractures and the proximity of the coronal fracture to the pulp as well as to serve as a reference point for future examinations. Pulpal testing (a Burton Vitalometer is an excellent instrument for this) should be performed to help determine the physiologic status of the pulp. A negative response should not be considered indicative of pulpal death at this time, since many teeth 
will not respond to testing until several weeks after an injury.

A simple enamel fracture may be treated by judicious stoning and polishing. A fracture involving dentine, however, should be covered with a temporary crown immediately, regardless of age, For this procedure the involved tooth may be isolated with a cotton roll and dried gently with warm air, the fractured surface wiped with eugenol, a layer of zinc oxideeugenol cement placed over the exposed dentine,

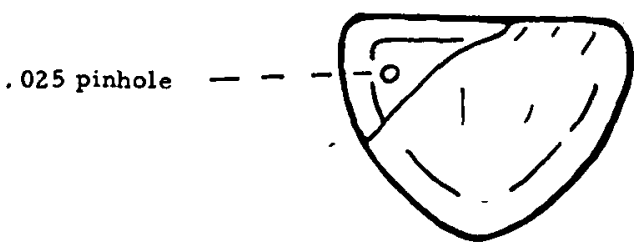

Fig. 1.-Site for pinhole to receive Markley wire.

and a preformed stainless steel crown or resin crown form trimmed to fit just beneath the free gingival tissue and cemented to the tooth. This temporary crown should be maintained until the final status of the pulp is ascertained. A minimal waiting period of four to six weeks is recommended before a final restoration is fabricated.

\section{Restorations}

The type of restoration used to restore a coronal fracture depends upon several factors: age and activity of the patient, extent of the fracture, colour of the tooth, size of the pulpal chamber, and occlusion.

(a) Acrylic restoration with pin. This restoration is usually selected when the fracture is small, involving little dentine, when there is no coronal discolouration, and when the pulp is vital. It may serve the patient through his early teens at which time a porcelain jacket or a gold-porcelain crown may be constructed. The preparation of the tooth is simple, very little tissue is removed, the labial surface is not involved, and the restoration produces good æsthetics. The acrylic, however, will change shade and the cavosurface margin is susceptible to decay due to the dimensional instability of the acrylic.

The tooth is prepared in the following fashion:

(1) Isolate with rubber dam.

(2) Remove unsupported enamel.

(3) Prepare a pin hole for $0.025^{\prime \prime}$ Markley wire with a Spirec 0.027" Bohrer bur. The pin hole should be $2.5 \mathrm{~mm}$. in depth and placed in the cervical area of the tooth just medial to the dento-enamel junction and slightly to the lingual (Fig. 1).

(4) Prepare retentive grooves where possible in dentine with a $1 / 2$ bur.

(5) Place a small ledge in the incisal portion of dentine with a 34 bur to support the free end of the Markley wire (Fig. 2).

(6) Cut the Markley wire long enough to accommodate for the right angle bend, place wire in pin hole and mark where the bend is to be.

(7) Bend the wire and cut its free end to proper length so it will fit snugly in the incisal ledge (Fig. 3).

(8) Mix zinc-phosphate cement to inlaysetting consistency and place in pin hole with lentulo spiral filling instrument. Place wire in pin hole and allow cement to harden.

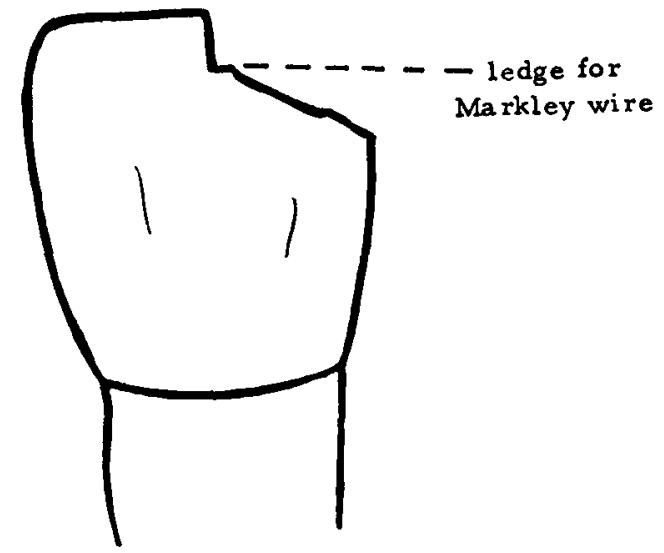

Fig. 2.-Ledge for transverse section of wire.

The acrylic is placed in the angle according to the following technique:

(1) Place a thin layer of Dycal over the exposed dentine.

(2) Paint cavity primer over the entire area to be covered with acrylic, wetting-not flooding-the surfaces.

(3) Place a resin matrix strip around the tooth and wedge, tacking down the free ends of the strip with compound on the adjacent teeth.

(4) Paint acrylic in the angle, waiting 10 seconds between each addition. After its insertion, cover the acrylic with a lubricant to prevent excessive drying by air and allow to set for a minimum of five minutes. 
(5) Remove matrix strip and finish margins and adjust contours with lubricated discs.

(b) Three pin three-quarter crown. The indications for this restoration are similar to those for the acrylic-pin restoration. The three-quarter crown is considerably more complex to fabricate. It will, however, provide greater support for the tooth when incisal forces are severe, and there is little likelihood of marginal leakage.

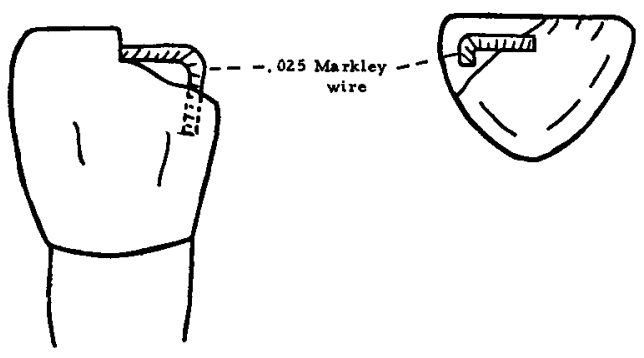

Fig. 3.-Location of wire on fractured tooth.

The tooth is prepared by:

(1) Slicing the proximal surface minimally to remove undercuts,

(2) Reducing the lingual enamel about the thickness of a sheet of 28 gauge wax,

(3) Placing a finishing line around the cingulum to connect the slices, and

(4) Placing three pin holes just pulpal to the dento-enamel junction-one in the cingulum and the other two on the lingual surface near the incisal edge but far enough away to avoid casting a shadow through the labial enamel when the restoration is seated; the two incisal pin holes should be about 1 millimetres deep, and the cingulum pin about $2 \frac{1}{2}$ millimetres deep; all pin holes should be finished with a size 700 bur and should be placed parallel to the long axis of the root.

An impression of the preparation may be secured either with compound or rubber base material. Plastic pins should be placed in the pin holes prior to taking the impression.

Medium-hard gold should be used for the casting to provide adequate incisal edge strength. The window area should be cut through to the lingual to reduce the metallic shadow and produce better esthetics. The window may be flled with silicate cement or acrylic after cementation of the crown with the shade selected carefully. Although the silicate cement will have to be replaced periodically, it is generally more colour stable than self-curing acrylic. The principal disadvantage to the three-quarter crown is its asthetic deficiencies. Although shades can be matched rather well, translucencies cannot, and usually there is a line of demarcation between the silicate cement and the remaining enamel. On the plus side, the restoration requires relatively little cutting of sound tissue and provides excellent retention.

(c) Porcelain veneer crown. This restoration, of the full-coverage type, is utilized when the degree of coronal fracture is moderate or deep and greater retention is required, when the crown is discoloured, or in any instance when optimal æsthetics are required. The age of the patient and the size of the pulpal chamber are not significant factors since only a moderate amount of sound tissue is removed during the preparation.

The tooth is prepared in the following manner:

(1) The proximal surfaces are sliced with a flat diamond disc and shoulders are established well beneath the free gingival margins,

(2) The labial enamel is removed just to the dento-enamel junction, and a shoulder (the width of a number 557 bur) is prepared well beneath the free gingival margin-connecting the proximal shoulders,

(3) The lingual enamel is reduced about the thickness of a sheet of 28 gauge wax and a finishing line is established well beneath the free gingival tissue around the cingulum and continued to bevel the entire shoulder, and

(4) The incisal length is reduced as required.

Following completion of the preparation, a custom-made temporary acrylic crown should be fabricated. It should be fitted precisely to the gingival margins of the preparation and cemented with zinc oxide-eugenol cement.

The impression and the relations are secured in the usual manner. The casting (use medium hard gold) will cover the entire tooth, including the gingival margins. The gold covering the labial and proximal surfaces must be very thin in order to provide adequate thickness for the porcelain. Porcelain is preferred to acrylic for the veneer because of its superior æsthetics, resistance to abrasion, and because it is tolerated better by the gingival tissue.

(d) Miscellaneous restorations. Porcelain or acrylic jackets may be substituted for the 
porcelain or acrylic veneer crowns. Acrylic jackets should be used in combination with gold copings in order to minimize the dimensional instability of the acrylic. The shoulderless acrylic jacket should be avoided because of its relatively poor marginal adaptation and the resulting irritation to the gingival tissues. Porcelain jackets are probably the most æsthetic type of full-coverage restorations. They are used when æsthetic needs are at a premium and when the depth of bite is favourable.

When the root canal of a fractured incisor has been filled and when the remaining coronal tissue is inadequate to support a restoration, then a post and core must be fabricated. The post should extend to about one-half the length of the root canal, and the core should replace the coronal dentine. After the root canal has been tapered to receive the post, a piece of plastic is trimmed to fit the canal loosely, and enough inlay wax is added to the plastic so that the post fits the canal accurately. The dentine core is waxed to the post and the resulting pattern is invested and cast in medium hard gold. After the finished post and core is cemented, the preparation of the tooth is completed so that it may receive a jacket or veneer crown.

\section{Management of pulpally involved teeth}

If the pulp has been exposed by an injury and is vital, it may be treated by capping or pulpotomy. These procedures are more successful if done before the root ends close. Studies done at the University of Michigan indicate a percentage of success in the range of 95 . If the pulp has degenerated or if the severity of the fracture dictates the need for a post, then a pulpectomy and root canal filling are required.

\section{Root fractures}

When a root fracture occurs in the apical onethird and the tooth is not mobile, no treatment is indicated. Usually the fracture will heal, and the pulp will remain vital. If the root fracture occurs in the coronal or middle one-third, the coronal segment usually is mobile and splinting is required. Normally the splint is maintained for about four weeks. If the fracture occurs in the area of the gingival crevice (coronal to the alveolar crest) healing will not take place. In this event the coronal segment is removed, the root canal is filled, and a post-type restoration is constructed. If the natural crown is otherwise intact, it may be used as the restoration and cemented to the post.

\section{Avulsed teeth}

A tooth that has been knocked out completely results in a traumatic experience for the child and his parents. The resulting space usually will close rapidly, and replacement is ordinarily difficult during the growing period. Replantation of the avulsed tooth is a good procedure. When a tooth is avulsed, the child should be seen by the dentist immediately. The parent should be instructed to wrap the tooth in a piece of gauze or clean handkerchief moistened with water and bring the child and the tooth to the office. Upon arrival, the child should be sedated with an appropriate drug and made comfortable. The tooth may be treated in the following manner:

(1) Wash the tooth gently with soap and water, leaving intact the remaining periodontal fibres,

(2) Wrap the tooth in sterile gauze saturated with normal saline solution and imbed the wrapped tooth in a block of compound to avoid handling it during the root canal procedures,

(3) Open the pulpal chamber with sterile burs, remove the pulp, and medicate and fll the canal with gutta percha points,

(4) Fill the lingual opening with zinc-phosphate cement, shorten the root by about $\frac{1}{16}$ of an inch and seal the gutta percha to the apex with a warm instrument, and

(5) Remove the tooth from the compound and place in a disinfecting solution. The tooth is ready for replantation. The lingual opening can be filled with silicate cement at a later date.

The site of the injury is anæsthetized and the tooth repositioned firmly into its original position. If the replantation is not performed promptly, the blood clot may have to be removed with a curette. The tooth must be splinted, or usually it will extrude and fail to reattach. There are several splints which are acceptable: (1) a self-curing acrylic splint cemented to the anterior teeth and adjusted to the patient's occlusion, and (2) a splint formed by cementing anterior orthodontic bands with edgewise brackets to the replanted 
tooth and the two adjacent teeth; an 0.030 steel wire is positioned in the brackets and ligated to place.

The child should be referred routinely to his physician for a tetanus booster. The parent should be advised to anticipate some oozing around the tooth for the first two or three days and directed to keep the area clean with moist sponges. The patient should be reexamined in 24 hours to check the position and the splint, and thereafter as often as required. Usually the tooth will become stable in about two weeks at which time the splint may be removed.

Ankylosis and root resorption are sequelle that should be expected and may appear promptly or after a number of years. Although data are not available, the average life expectancy of a replanted tooth is probably about five years. In most instances this period will be long enough to permit the dentition to complete its development, after which replacement is less complex. A few replanted teeth have survived for more than 20 years.

\section{Summary}

Devices to prevent injuries to anterior teeth have been designed which are easy to wear and also allow reasonably good speech. A technique to construct an acceptable mouth protector has been described. A variety of injuries to primary and young permanent teeth have been discussed along with methods to treat them. Injured teeth should be examined and treated as soon as possible, and the outdated custom of postponing the restoration of a fractured incisor until some arbitrary age should be discarded.

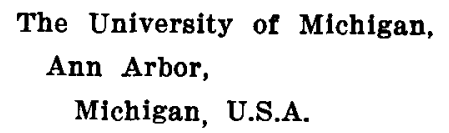

Meander - The sinuosity of river channels is clearly apparent in maps and aerial photographs, where the successive curves of a river often appear to have a certain regularity. In many instances the repeating patterns of curves is so pronounced that it is the most distinctive characteristic of the river. Such curves are called meanders after a winding stream in Turkey known in ancient Greek times as the Maiandros and to-day as the Menderes.-L. B. Leopold and W. B. Langbien, Scientific American, June, 1966. 